\title{
Topological effects and particle-physics analogies beyond the massless Dirac-Weyl fermion in graphene nanorings
}

\author{
Igor Romanovsky ${ }^{*}$ Constantine Yannouleas $\dagger^{\dagger}$ and Uzi Landman ${ }^{\dagger}$ \\ School of Physics, Georgia Institute of Technology, Atlanta, Georgia 30332-0430
}

(Dated: 30 December 2012; Phys. Rev. B 87, 165431 (2013))

\begin{abstract}
Armchair and zigzag edge terminations in planar hexagonal and trigonal graphene nanorings are shown to underlie one-dimensional topological states associated with distinctive energy gaps and patterns (e.g., linear dispersion of the energy of an hexagonal ring with an armchair termination versus parabolic dispersion for a zigzag terminated one) in the bands of the tight-binding spectra as a function of the magnetic field. A relativistic Dirac-Kronig-Penney model analysis of the tightbinding Aharonov-Bohm behavior reveals that the graphene quasiparticle in an armchair hexagonal ring is a condensed-matter realization of an ultrarelativistic fermion with a position-dependent mass term, akin to the zero-energy fermionic solitons with fractional charge familiar from quantum field theory and from the theory of polyacetylene. The topological origins of the above behavior are highlighted by contrasting it with the case of a trigonal armchair ring, where we find that the quasiparticle excitations behave as familiar Dirac fermions with a constant mass. Furthermore, the spectra of a zigzag hexagonal ring correspond to the low-kinetic-energy nonrelativistic regime of a leptonlike massive fermion. A onedimensional relativistic Lagrangian formalism coupling a fermionic and a scalar bosonic field via a Yukawa interaction, in conjunction with the breaking of the $Z_{2}$ reflectional symmetry of the scalar field, is shown to unify the above dissimilar behaviors.
\end{abstract}

PACS numbers: 73.22.Pr, 73.21.-b, 11.10.-z, 73.23.Ra

\section{INTRODUCTION}

Since its inception, relativistic quantum mechanics has been associated mainly with the fields of particle and high-energy physics ${ }^{1}[4$ Recently, however, a tabletop version of relativistic quantum physics emerged, following the experimental isolation of graphene, which is a single-layer, planar honeycomb lattice of carbon atoms. Indeed, the two-dimensional (2D) quasiparticle excitations of neutral graphene near the Fermi level behave $e^{6 / 7}$ as massless neutrinolike fermions described by the celebrated Dirac-Weyl ${ }^{8}$ (DW) equation. The scientific and technological potential for exploiting charge carriers and quasiparticles with relativistic behavior in tunable condensed-matter and atomic-physics systems is attracting much attention. ${ }^{9}[13$ In this context, an important question, as yet only partly explored, remains whether quasi-onedimensional (1D) graphene systems support exclusively DW massless or constant-mass Dirac fermions, or they can induce relativistic-quantum-field (RQF) behaviors that require consideration of position-dependent mass terms, reflecting generalized underlying bosonic scalar fields.

In this paper, we show that planar graphene nanorings do indeed exhibit a rich variety of physics, ranging from sophisticated RQF regimes to more familiar cases of constant-mass fermions (both in the relativistic and nonrelativistic regimes). The emergence of these physical regimes depends on the specific combination of topological factors associated with modifications of the graphene lattice, such as the type of edge termination (i.e., armchair or zigzag) and the shape (i.e., hexagonal or trigonal) of the graphene ring.

To this end, we investigate the properties of the cor- responding tight-binding $7 / 14 \mid 15$ (TB) spectra and of the associated Aharonov-Bohm ${ }^{16}$ (the AB effect, which is a hallmark topological effect in condensed matter systems) oscillations of the magnetization, as a function of the magnetic field $B$. We then analyze the spectra and $A B$ characteristics with the help of a Dirac-Kronig-Penney (DKP) superlattice model, $\stackrel{2[17}{2}$ in the spirit of the virtual "band-structure" model for the nonrelativistic $\mathrm{AB}$ effect $\frac{18}{18}$ We find that the relativistic behavior in armchair rings requires a profound modification of the $1 \mathrm{D}$ Dirac equation ${ }^{1}$ through the introduction of a positiondependent mass term, in analogy with the fractionalcharge, zero-energy topological modes in quantum field theory and in the theory of trans-polyacetylene ${ }^{1922} \mathrm{In}$ contrast, the zigzag-ring spectra may correspond to the low-energy nonrelativistic regime of a leptonlike ${ }^{23}$ massive particle, heavier than the electron.

Planar graphene rings (and the associated AB spectra) have been recently investigated by a number of groups using tight-binding $\frac{15124}{28}$ methods (for polygonal shapes with armchair or zigzag terminations), as well as continuum DW ${ }^{24 / 29 / 30}$ equations supplemented with infinitemass boundary conditions (for idealized circular shapes). These earlier studies did not address the question of possible analogies to 1D quantum field theoretical models and particle physics. We stress that a prerequisite to raising and answering this question is the introduction by us of the virtual DKP superlattice model for the $\mathrm{AB}$ effect. For a review on recent experimental studies of the Aharonov-Bohm effect in graphene nanorings, see Ref. 31 .

In addition to planar graphene rings, graphene nanoribbons (GNRs) are another class of related quasi1D systems. GNRs have attracted substantially more 
attention than graphene rings and their study gave rise to a vast body of theoretica $2^{32 \sqrt[35]{35}}$ and experimenta $a^{\sqrt[36]{3}}$ literature. For graphene nanoribbons, it was found that a gap $\Delta_{0}$ may open at the Fermi energy, leading to an apparent analogy with the constant-mass Dirac fermion [see Ref. 32(c)]. As elaborated below, for an armchair nanoribbon (aGNR), this gap arises from the topology of the armchair edge which mimicks the dimerized domains (i.e., formation of Kekulé unequal carbon bonds) in trans-polyacetylene ${ }^{1922}$ Then in analogy with the scalar $Z_{2}$ kink-soliton associated with the Peierls transition in trans-polyacetylene (or equivalently with the $Z_{2}$ kink-soliton used in the Jackiw-Rebbi fermionic RQF mode ${ }^{37}$ ) the qualitative features of the (fermionic) $\mathrm{AB}$ spectra of armchair graphene rings can be understood as resulting from an alternation (or lack of it) of two degenerate dimerized domains associated with the arms of the graphene ring. We stress that the effective dimerization in the armchair GNRs and armchair graphene nanorings has a topological origin imposed by the presence of the armchair edges, while the dimerization in transpolyacetylene is due to the Peierls instability ${ }^{21 / 38}$ These two different underlying processes, however, lead to similar results that are characterized by the breaking of the $1 \mathrm{D} Z_{2}$ reflectional symmetry (see in particular Secs. IV A and $\mathrm{V}$ below).

Our findings of quasiparticles in graphene with general position-dependent, and/or constant (rest), masses (unlike the massless neutrinolike quasiparticle in 2D graphene) is particularly interesting in light of increasing current interest in mass acquisition mechanisms, e.g., the Higgs mechanism in elementary particles ${ }^{39} 41$ and condensed-matter physics $\underline{42143}$

The predicted unprecedented emergent unfolding of fundamentally distinct physical regimes, namely complex quantum-field theoretical ones versus nonrelativistic constant-mass ones, depending solely on the materials' shape and edge termination is to date unique to graphene. It will be of great interest to test signatures of such regime-crossover experimentally for specifically prepared graphene systems with atomic precision, $\underline{36}$ as well as to explore possible occurrence of such topological-in-origin physical behavior in other designerDirac-fermion artificial systems ${ }^{9 / 12}$ or nanopatterned artificial graphene. $\frac{44}{4}$

The plan of the paper is as follows.

Sec. II describes the Aharonov-Bohm tight-binding spectra for three characteristic planar graphene nanorings, i.e., an armchair hexagonal ring, an armchair trigonal ring, and a zigzag hexagonal ring.

Sec. III introduces the theoretical aspects of a relativistic 1D Dirac-Kronig-Penney model, based on the generalized Dirac equation. The DKP model describes the virtual superlattice associated with the AharonovBohm effect.

Sec. IV presents the DKP interpretation of the tightbinding spectra calculated in Sec. II The quasiparticle excitations in graphene nanorings are shown to exhibit behavior associated with quantum field theoretical models for elementary particles beyond the massless DiracWeyl fermion.

Sec. V discusses the full relativistic quantum field Lagrangian formalism that underlies the DKP interpretation elaborated in Sec. IV] The Lagrangian formalism shows that the physics of quasiparticle excitations in planar graphene nanorings relates to mass acquisition and formation of fermionic solitons.

Finally, Sec. VI presents our conclusions.

\section{TIGHT-BINDING CALCULATIONS}

In this section, we will describe the TB spectra and corresponding $\mathrm{AB}$ magnetizations (as a function of the magnetic flux) for three characteristic cases of planar graphene rings, and specifically for a hexagonal armchair ring, a trigonal armchair ring, and a hexagonal zigzag ring (all three of similar dimensions). We note that the arms of the armchair rings studied here correspond to the class of perfect armchair nanoribbons referred to as metallic, 32 (b) 33 35 they exhibit a vanishing energy gap, $\Delta_{0}=0$, between the valence and conduction bands in tight-binding and continuum DW calculations. However, any perturbation (including the incorporation into a nanoring structure) may result ${ }^{32(\mathrm{~b})}$ in the opening of a gap $\Delta_{0}>0$. The metallic aGNRs have a width corresponding to $\mathcal{N}_{W}=3 l-1, l=1,2,3, \ldots$ carbon atoms. Counting along a zigzag line in the middle of the arm (away from the corners), the hexagonal and trigonal armchair rings studied in this paper $\frac{\sqrt{45}}{}$ have $\mathcal{N}_{W}=14$.

To determine the single-particle spectrum [the energy levels $\left.\varepsilon_{i}(B)\right]$ in the tight-binding calculations for the graphene nanorings, we use the hamiltonian

$$
H_{\mathrm{TB}}=-\sum_{<i, j>} \tilde{t}_{i j} c_{i}^{\dagger} c_{j}+h . c .
$$

with $<>$ indicating summation over the nearest-neighbor sites $i, j$. The hopping matrix element

$$
\tilde{t}_{i j}=t_{i j} \exp \left(\frac{i e}{\hbar c} \int_{\mathbf{r}_{i}}^{\mathbf{r}_{j}} d \mathbf{s} \cdot \mathbf{A}(\mathbf{r})\right)
$$

where $\mathbf{r}_{i}$ and $\mathbf{r}_{j}$ are the positions of the carbon atoms $i$ and $j$, respectively, and $\mathbf{A}$ is the vector potential associated with the applied constant magnetic field $B$ applied perpendicular to the plane of the nanoring.

The $\mathrm{AB}$ magnetization of the graphene ring is given by

$$
M(\Phi)=-S \frac{d E_{\mathrm{tot}}}{d \Phi}
$$

where the total energy

$$
E_{\mathrm{tot}}(\Phi)=\sum_{i, \sigma}^{\mathrm{occ}} \varepsilon_{i}(\Phi)
$$


(a)

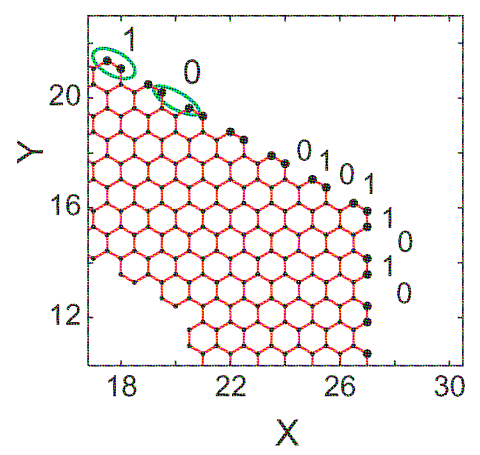

(b)

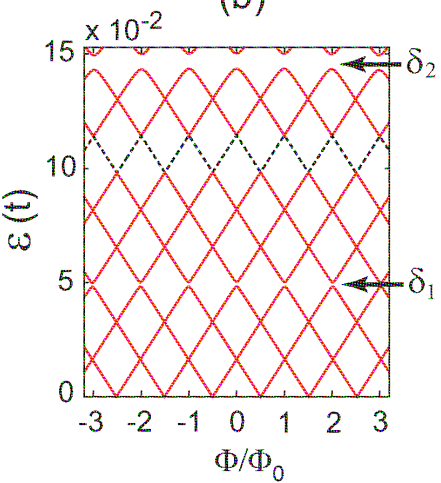

(c)

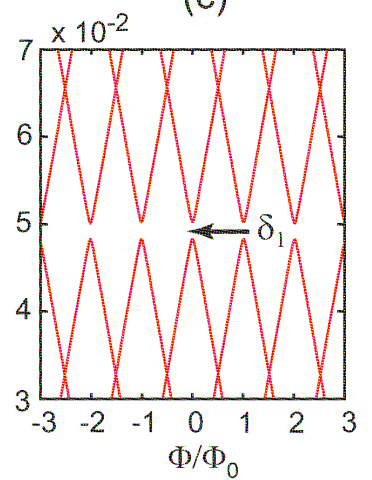

FIG. 1. (Color online) (a) Part of the hexagonal graphene ring (2718 carbon atoms) with armchair edges. 1 and 0 denote the short and long carbon dimers, respectively. Lengths in units of the graphene lattice constant $a_{0}=0.246 \mathrm{~nm}$. (b) TB single-particle spectrum as a function of the magnetic flux (magnetic field). Energies in units of the TB hopping-parameter $t=2.7 \mathrm{eV}$. The dashed black line denotes the Fermi level for $N=14$ electrons. The arrows highlight the band gaps. (c) Magnification of the TB spectrum around the $\delta_{1}$ band gap. $\delta_{1} \sim 80 \mathrm{~K}$, and thus it is easily detectable experimentally.

(a)
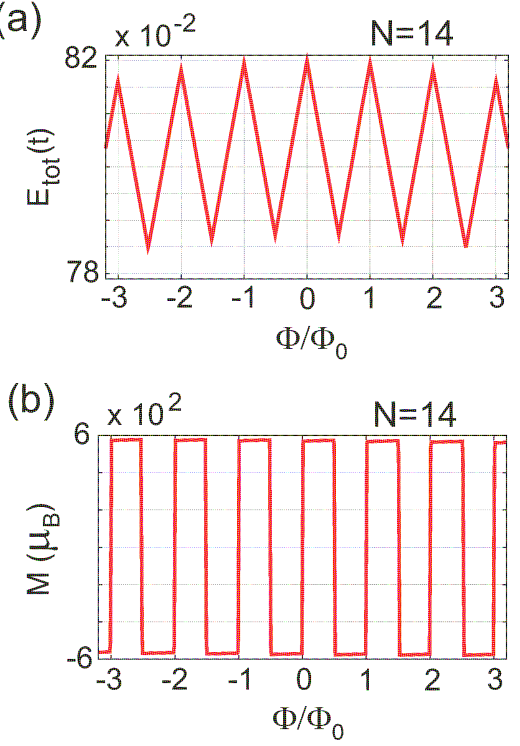

FIG. 2. (Color online) (a) TB total energies (sum over singleparticle energies including spin) for $N=14$ quasiparticles. (b) Correposnding TB magnetization (in units of the Bohr magneton). Energies in units of the TB hopping-parameter $t=2.7 \mathrm{eV}$.

is given by the sum over all occupied single-particle energies; the index $\sigma$ runs over spins. $\Phi=B S$ is the magnetic flux through the area $S$ of the graphene ring and $\Phi_{0}=h c / e$ is the flux quantum.

The diagonalization of the TB hamiltonian [Eq. (1)] is implemented with the use of the sparse-matrix solver ARPACK ${ }^{46}$ In calculating $E_{\text {tot }}$ [see Eq. (40], only the single-particle TB energies with $\varepsilon_{i}(B)>0$ are considered 24

The TB results exhibit significant differences between the three cases studied here. These differences fall into two categories, namely, (A) same edge termination but different shape and (B) same shape but different edge termination.

\section{A. Hexagonal versus trigonal armchair rings}

The shape of the hexagonal graphene ring with armchair edge terminations considered here, as well as the corresponding TB results regarding the single-particle spectrum, the total energy, and the magnetization are displayed in Fig. 1 and Fig. 2, as a function of $\Phi / \Phi_{0}$. The shape of the trigonal graphene ring with armchair edge terminations considered here, as well as the corresponding TB results regarding the single-particle spectrum are displayed in Fig. 3 .

Both TB energy spectra in Fig. 1(b) (armchair hexagon) and Fig. 3(c) (armchair triangle) are organized in braid bands separated by energy gaps. They exhibit, however, two main differences. The first concerns the composition of the braid bands, with the hexagonal ring exhibiting six-membered bands while the trigonal ring having three-membered bands. The sixfold and threefold groupings are a reflection of the $Z_{6}$ and $Z_{3}$ point-group symmetry of the hexagonal and trigonal shapes, respectively; these symmetries are fully taken into account by the DKP modeling in Sec. III.

The second important difference between the TB energy spectra in Fig. 1(b) and Fig. 3(c) concerns the number and nature of energy gaps. Specifically, two regular superlattice gaps $\delta_{1}$ and $\delta_{2}$ are present in both cases; note the similar energy scale [a magnification of the region around the $\delta_{1}$ gap is shown in Fig. 11(c)]. However, while a mass gap $\Delta_{0}$ is well developed for the trigonal ring [Fig. 3(c)], no corresponding $\Delta_{0}$ gap is present in the spectrum of the hexagonal ring, where the $\varepsilon=0$ 

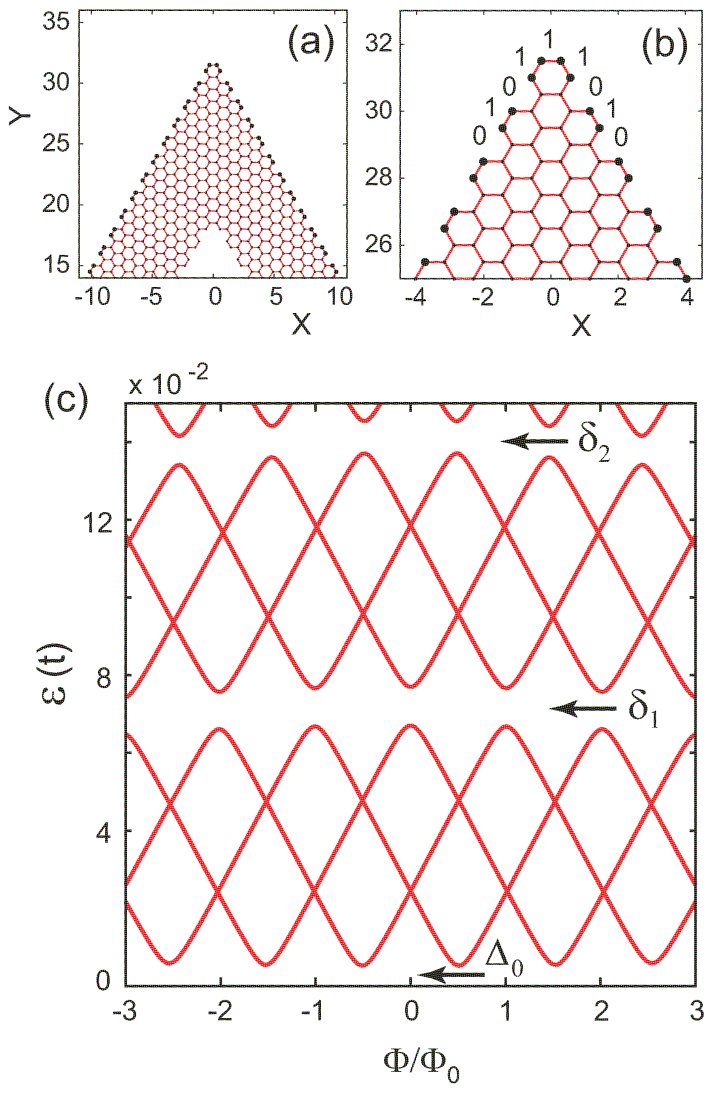

FIG. 3. (Color online) (a) Part of the trigonal graphene ring (2142 carbon atoms) with armchair edges. (b) Magnification of the corner of the trigonal ring shown in (a). 1 and 0 denote the short and long carbon dimers, respectively. Lengths in units of the graphene lattice constant $a_{0}=0.246 \mathrm{~nm}$. (c) TB single-particle spectrum as a function of the magnetic flux (magnetic field). Energies in units of the TB hoppingparameter $t=2.7 \mathrm{eV}$. The two arrows denoted $\delta_{1}$ and $\delta_{2}$ highlight band gaps. The arrow denoted by $\Delta_{0}$ indicates the opening of a gap at the Fermi level $(\varepsilon=0)$ associated with generation of a rest mass $\mathcal{M}$. Note that the $\Delta_{0}$ gap is absent in the TB spectrum of the hexagonal graphene ring in Fig. 1 (b).

horizontal axis dissects (splits in half) the corresponding sixfold braid band [Fig. [1(b)]. As we will show in Sec. IV] the gap $\Delta_{0}$, for the case of the armchair triangle, is consistent with the physics of a massive (but still relativistic) Dirac fermion, while the dissecting of the $\varepsilon=0$ sixfold band, in the case of the armchair hexagon, is consistent with the formation of a fermionic soliton $\frac{37}{37}$ built on a scalar $Z_{2}$ kink soliton (precisely, a train of six fermionic solitons attached to successive $Z_{2}$ kink/antikink solitons; see also Sec. VP.

\section{B. Armchair versus zigzag hexagonal rings}

As aforementioned, the TB results exhibit also significant differences between the cases of hexagonal rings with
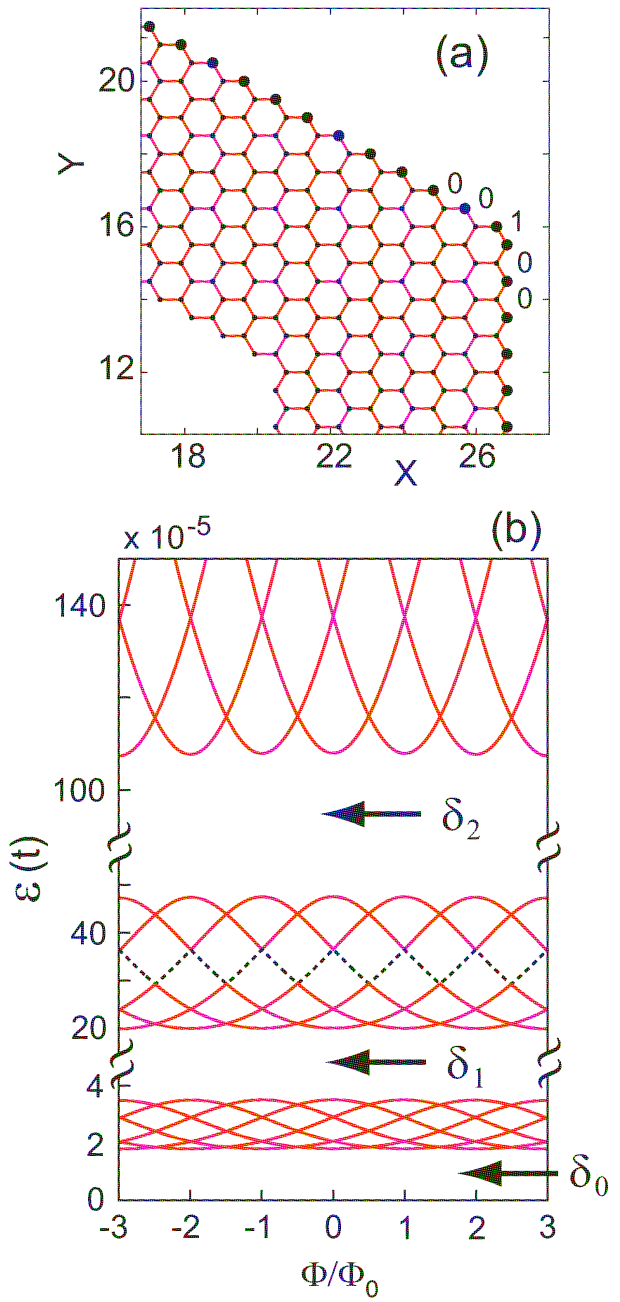

FIG. 4. (Color online) (a) Part of the hexagonal graphene ring (2688 carbon atoms) with zigzag edges. 1 and 0 denote the short and long carbon dimers, respectively. Lengths in units of the graphene lattice constant $a_{0}=0.246 \mathrm{~nm}$. (b) TB singleparticle spectrum as a function of the magnetic flux (magnetic field). Energies in units of the TB hopping-parameter $t=2.7$ $\mathrm{eV}$. The dashed black line denotes the Fermi level for $N=20$ quasiparticles. The arrows highlight the band gaps.

armchair and zigzag terminations. One such difference concerns the $B$-dependence of the single-particle energies $\varepsilon(\Phi)$, which is piecewise linear for the armchair case [Fig. 1(b)], but piecewise parabolic for the zigzag case [Fig. 4(b)]; this maintains also in the total energies $E_{\text {tot }}(\Phi)$ [Fig. 2(a) and Fig. 5(a)]. For the AB magnetizations [see Eq. [30], this results in a characteristically different profile for the $\mathrm{AB}$ oscillations of $M(\Phi)$ : step-staggeredlike in the armchair case [Fig. 2(b)] and sawtoothlike [Fig. 5 (b)] in the zigzag case. The paraboliclike $B$-dependence in the zigzag edge case ${ }^{15}$ is reminiscent of the spectra of a nonrelativistic ideal metal ring ${ }^{47}$ In contrast (see below), the linear $B$-dependence (in conjunction with the other features of the hexagonal armchair spectrum) can be associated with the fully relativistic regime of Dirac 

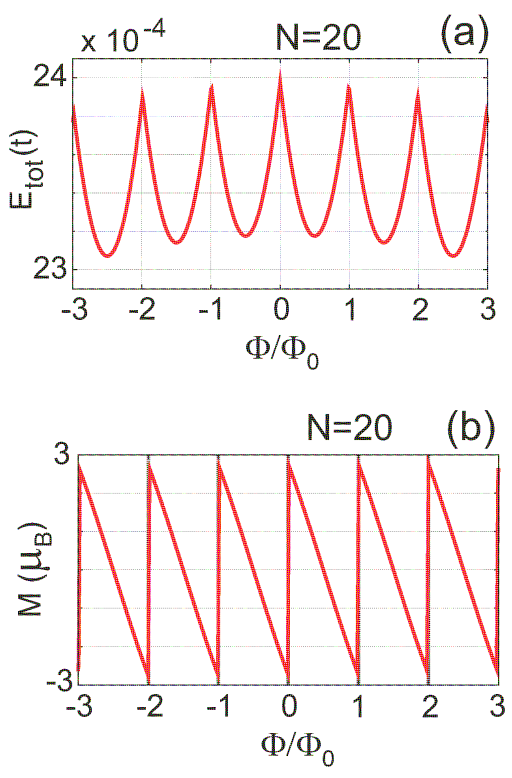

FIG. 5. (Color online) (a) TB total energies (sum over singleparticle energies including spin) for $N=20$ quasiparticles. (b) Correposnding TB magnetization (in units of the Bohr magneton). Energies in units of the TB hopping-parameter $t=2.7 \mathrm{eV}$.

fermions with position-dependent masses 19120

Both the armchair and zigzag single-particle spectra for hexagonal are organized in six-member braid bands separated by energy gaps. This sixfold grouping is a reflection of the $Z_{6}$ point-group symmetry of the hexagonal rings. The energy gaps in the zigzag case are comparable to the width of the braid bands, and both the gaps and the widths of the bands increase with higher energy [see Fig. 4(b)]; this is consistent with a nonrelativistic Kronig-Penney model ${ }^{14}$ In Fig. 4 (b), there are three energy gaps labeled as $\delta_{0}, \delta_{1}$, and $\delta_{2}$. Unlike the relativistic regime, in the nonrelativistic limit a gap around $\varepsilon=0$ is unrelated to the particle mass, and for this reason we use the symbol $\delta_{0}$ (with a lower-case $\delta$ ) instead of $\Delta_{0}$ as was the case in Fig. 3(c). We note that in the nonrelativistic regime the effective mass of the quasiparticle excitations is proportional to the inverse of the second derivative of the (approximately) parabolic spectra; see, e.g., Ref. 48.

\section{DIRAC-KRONIG-PENNEY SUPERLATTICE}

As was shown for a semiconductor ring using a nonrelativistic superlattice approach, the $\mathrm{AB}$ single-particle spectrum exhibits energy gaps demarcating Bloch bands when a scatterer is placed on the ring 18 In this context, the energy gaps that appear in Fig. 1(b), Fig. 33(c), and Fig. 4(b) indicate that the $\mathrm{AB}$ effect in polygonal graphene rings should be analyzed and modeled with the help of 1D Kronig-Penney-type superlattices, with the corners of the polygons providing a generalized analog to the "scatterers" of Ref. 18. Specifically, we consider a $1 \mathrm{D}$ relativistic Dirac-Kronig-Penney model with unit cells built out of square potential barriers (developed in Ref. 2] in the context of the physics of quarks).

The DKP model considered here is based on the 1D generalized Dirac equation, which has the form:

$$
[E-V(x)] I \Psi+i \hbar v_{F} \alpha \frac{\partial \Psi}{\partial x}-\beta \phi(x) \Psi=0,
$$

with $v_{F}$ being the Fermi velocity of graphene, which replaces the speed of light $c ; v_{F} / c \approx 1 / 300 . V(x)$ is an electrostatic potential and $\phi(x)$ is a bosonic positiondependent scalar field. We note that Ref. 2 uses $\phi(x) \equiv$ $\mathcal{M} v_{F}^{2}+V_{s}(x)$, with $\mathcal{M}$ denoting the rest mass of a Dirac fermion (including the massless case) and the term $V_{s}(x)$ being referred to as the Lorentz scalar potential. Omitting the last term on the left of Eq. (5) reduces this equation to the massless Dirac-Wey $\sqrt{8}$ one that underlies the majority of studies in planar graphene.

The fermion field $\Psi$ is a twodimensional vector

$$
\Psi=\left(\begin{array}{c}
\psi_{u} \\
\psi_{l}
\end{array}\right)
$$

where the subscripts $u$ and $l$ stand for the upper and lower component, respectively. The $2 \times 2$ Dirac matrices $\alpha$ and $\beta$ can be $e^{49}$ any two of the three Pauli matrices

$$
\sigma_{1}=\left(\begin{array}{cc}
0 & 1 \\
1 & 0
\end{array}\right) ; \sigma_{2}=\left(\begin{array}{cc}
0 & -i \\
i & 0
\end{array}\right) ; \sigma_{3}=\left(\begin{array}{cc}
1 & 0 \\
0 & -1
\end{array}\right) \text {. }
$$

For example in the Dirac representation, one has $\alpha^{D}=$ $\sigma_{1}$ and $\beta^{D}=\sigma_{3} . \quad I$ is the $2 \times 2$ identity matrix. We stress that the energy spectra of the DKP model are independent of the specific representation used for $\alpha$ and $\beta$. Below we will often use the notation $m(x)$, instead of $\phi(x)$, to stress the fact that $\phi(x)$ can be considered as a position-dependent mass term.

In the DKP modeling of the TB results, we assign to the $n$th side $\left(n=1, \ldots, N_{s}\right)$ of the polygonal ring a number $(J)$ of square potential steps (regions) denoted as $\left(V_{i}^{(n)}, m_{i}^{(n)}\right), i=1, \ldots, J$. We note again that the electrostatic potentials $V_{i}^{(n)}$ enter the 1D Dirac equation [Eq. 5 through the energy term $(E-V) I \Psi$, while the mass terms $m_{i}^{(n)}$ replace the scalar potential in the term $\beta \phi(x) \Psi$; as a result these two potentials lead to different physical behavior in the relativistic regime.

The building block of the DKP model is a $2 \times 2$ wavefunction matrix $\boldsymbol{\Omega}$ formed by the components of two independent $2 \times 1$ spinor solutions of the onedimensional firstorder Dirac equation. $\boldsymbol{\Omega}$ plays ${ }^{2}$ the role of the Wronskian matrix $\mathbf{W}^{50}$ used in the second-order nonrelativistic KP model; it is defined as follows at a point $x$ of the unit cell (here we use the Dirac representation):

$$
\boldsymbol{\Omega}_{K}(x)=\left(\begin{array}{cc}
e^{i K x} & e^{-i K x} \\
\Lambda e^{i K x} & -\Lambda e^{-i K x}
\end{array}\right)
$$




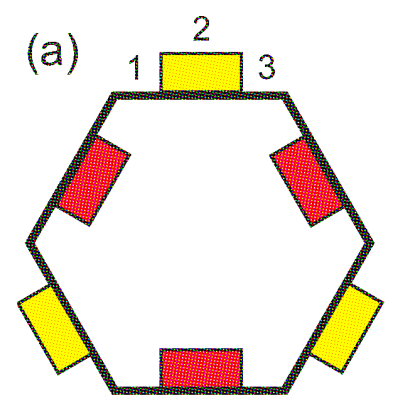

armchair

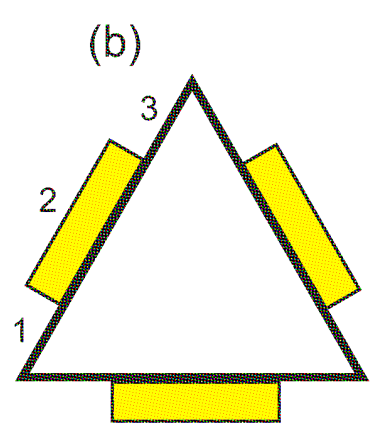

armchair

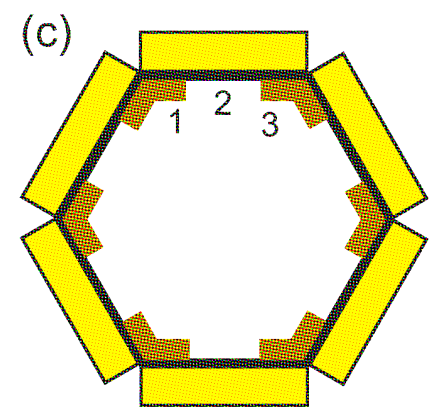

zigzag

FIG. 6. (Color online) Schematic representation of the $\left(V_{i}^{(n)}, m_{i}^{(n)}\right), i=1, \ldots, 3$ and $n=1, \ldots, N_{s}$ square-step parameters entering in the DKP calculations: (a) in Sec. IV A. (b) in Sec. IVB. (c) in Sec. IV C Each side of the polygon is divided in three lengths $L_{1}, L_{2}$, and $L_{3}$. Boxes outside a polygon (online yellow) represent values $m_{0}>0$. Boxes inside a polygon (online red) represent values $-m_{0}<0$. The nonzero values for the $V_{1}^{(n)}$ and $V_{3}^{(n)}$ in (c) are portrayed by thick lines (online brown) in the interior of the schematic hexagon. Zero values of parameters are not highlighted.

where

$$
K^{2}=\frac{(E-V)^{2}-m^{2} v_{F}^{4}}{\hbar^{2} v_{F}^{2}}, \quad \Lambda=\frac{\hbar v_{F} K}{E-V+m v_{F}^{2}} .
$$

We note again that, unlike the case of the original Dirac equation, $\frac{1}{1} m$ here is not a constant, but it may take different values from one region to the next. The transfer matrix for a given region (extending between two matching points $x_{1}$ and $\left.x_{2}\right)$ is the product $\mathbf{M}_{K}\left(x_{1}, x_{2}\right)=$ $\boldsymbol{\Omega}_{K}\left(x_{2}\right) \boldsymbol{\Omega}_{K}^{-1}\left(x_{1}\right)$; this latter matrix depends only on the width $x_{2}-x_{1}$ of the region, and not separately on $x_{1}$ or $x_{2}$. The relativistic $\mathbf{M}$ matrices defined here correspond to those considered ${ }^{\sqrt{51}}$ in the case of a nonrelativistic superlattice in Ref. 14 .

The transfer matrix corresponding to the $n$th side of the hexagon is the product

$$
\mathbf{t}_{n}=\prod_{i=1, J} \mathbf{M}_{K}\left(x_{i}, x_{i+1}\right), \quad x_{1}=0, x_{J+1}=L,
$$

with $L$ being the (common) length on the hexagon side. The transfer matrix associated with the complete unit cell (around the ring) is the product

$$
\mathbf{T}=\prod_{n=1}^{N_{s}} \mathbf{t}_{n},
$$

where $N_{s}$ is the number of sides of the polygonal shape considered $\left(N_{s}=3\right.$ and $N_{s}=6$ for a triangle and hexagon, respectively).

Following Ref. 18, we consider the supperlattice generated from the virtual periodic translation of the unit cell as a result of the application of a magnetic field $B$ perpendicular to the ring. Then the $\mathrm{AB}$ energy spectra are given as solutions of the dispersion equation

$$
\cos \left[2 \pi\left(\Phi / \Phi_{0}+\eta\right)\right]=\operatorname{Tr}[\mathbf{T}(E)] / 2,
$$

where we have explicitly denoted the dependence of the r.h.s. on the energy $E$. The presence $(\eta=1 / 2)$ or absence $(\eta=0)$ of an additional flux-shift in the relativistic or nonrelativistic case, respectively, follows through a comparison of the patterns of $\mathrm{AB}$ oscillations of a Dirac/Schrödinger electron in the limiting case of an ideal metallic circular ring. $47 / 52$

\section{DKP INTERPRETATION OF TIGHT-BINDING CASE STUDIES}

In this section, we demonstrate that our DKP modeling can capture the essential physics underlying the TB spectra in Fig. 1(b), Fig. 3(c), and Fig. 4(b). In this respect, it also provides a framework for unifying the broad variety of behaviors of the TB spectra of graphene nanorings. A schematic representation of the parameter sets used in our DKP simulations is given in Fig. 6 .

\section{A. Armchair hexagonal ring and position-dependent-mass relativistic regime}

First we attempt a solution corresponding to the generalized Dirac equation (5) with $\phi(x)=0$. In Fig. 7(a), we display the DKP spectra of a massless excitation (i.e., $m_{i}^{(n)}=0$ for all $i$ and $\left.n=1, \ldots, 6\right)$. This massless DKP spectrum does not exhibit any gaps and it is strictly linear and periodic (with period $\Phi_{0}$ ) as a function of $\Phi$; this correlates with the behavior of a free massless fermion, as in the case of $2 \mathrm{D}$ graphene. We note that this gapless spectrum remains unchanged even when we consider in addition electrostatic potential steps, $V_{i}^{(n)}>0$, a fact that is a reflection of Klein tunneling. 53.54

However, the TB spectrum in Fig. 1(b) exhibits energy 
(a)

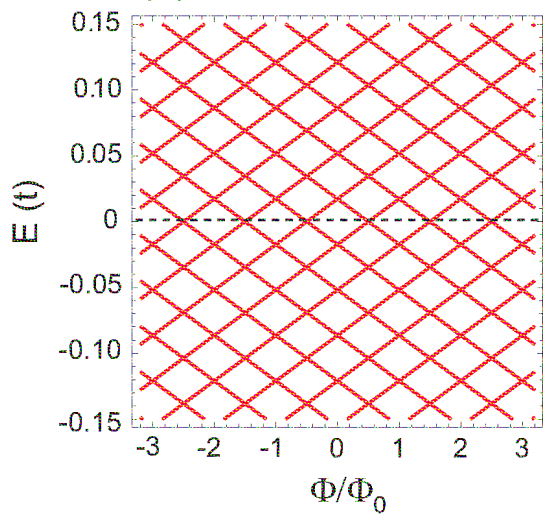

(b)

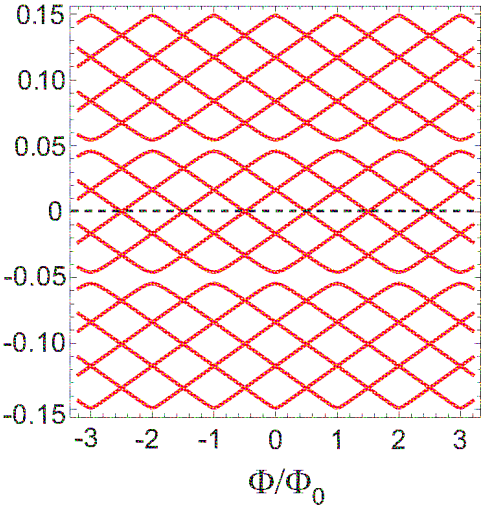

(c)

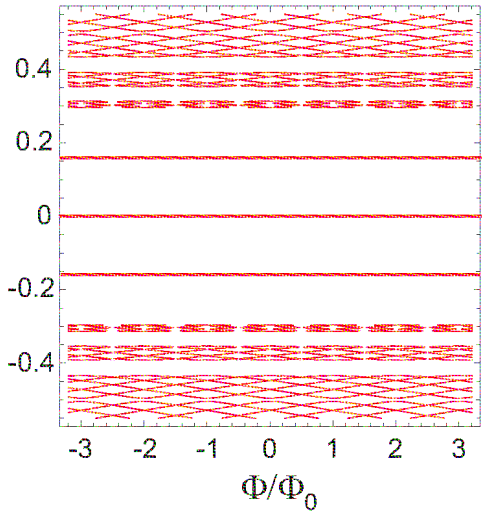

FIG. 7. (Color online) Spectra from the DKP model (relativistic regime) corresponding to the schematic case (hexagon) in Fig. 6(a); see text and Eqs. 13 15 for the full set of parameters employed. (a) $m_{0}=0$ and any $V_{i}$. Note the absence of band gaps due to the Klein paradox. (b) $m_{0}=0.01 t / v_{F}^{2}$. Note the similarity of the spectrum with that of the armchair graphene ring in Fig. 1 (b). (c) $m_{0}=0.30 t / v_{F}^{2}$. The horizontal lines result from suppression of the $\mathrm{AB}$ oscillations due to strong localization. The dashed line indicates the energy zero.

gaps (denoted as $\delta_{1}$ and $\delta_{2}$, and highlighted by arrows), which require consideration of potential barriers in the DKP modeling. In the spirit of earlier investigations of real-space superlattices in 2D graphene, ${ }^{[5]}[57$ we consider first a constant mass $m_{i}^{(n)}=\mathcal{M}>0$, and alternating $V_{i}^{(n)}=V_{0}>0$ and $V_{i}^{(n)}=0$ steps in consecutive regions (see Sec. III). However, calculations with this choice show an opening of an energy gap at $\varepsilon=0$, a fact that conflicts with Fig. 1(b); in addition, it does not preserve the particle-hole symmetry of the TB spectra.

A crucial feature of the TB armchair spectra in Fig. 1.(b) is the presence of zero-energy states (at half-integer fluxes). In order to capture this feature, and in light of our failed choices (see above), we attempt next to use a non-vanishing position-dependent scalar field $\phi(x)$ [denoted also as $m(x)$ ] in Eq. (5), and set the electrostatic potential $V(x)=0$. Recalling certain key elements in the theory of trans-polyacetylene pertaining to zero-energy solitonic modes, $\frac{19 \mid 20}{20}$ we employ in our DKP transfermatrix solution of Eq. (5) a scalar potential $\phi(x)$ of the form $m(x)=-m(-x)$. Consequently, we divide each side of the hexagon in three parts $(J=3)$ of length

$$
L_{1}^{(n)}=a, \quad L_{2}^{(n)}=b, \quad L_{3}^{(n)}=a,
$$

and assign values

$$
V_{1}^{(n)}=V_{2}^{(n)}=V_{3}^{(n)}=0,
$$

and

$$
m_{1}^{(n)}=m_{3}^{(n)}=0, \quad m_{2}^{(n)}=(-1)^{n} m_{0} .
$$

Note that the index $n=1, \ldots, 6$ here is numbering the sides of the hexagon, and thus overall the positiondependent mass term in our model is antisymmetric around each corner of the haxagonal ring. A schematic representation of the above parameters [Eq. (13) - Eq. [15p] is given in Fig. 6(a).

Fig. 7(b) displays the DKP spectrum calculated with the dispersion equation $(12)$ using the above parameter set with $m_{0}=0.01 t / v_{F}^{2}$, and $a=8 a_{0}, b=15 a_{0}$ One observes that, in addition to the piecewise linear $B$-dependence, the DKP spectrum faithfully reproduces the two other central features of the TB spectrum [Fig. 1(b)]: (i) the zero-energy states at half-integer values of $\Phi / \Phi_{0}$ and (ii) the opening at higher energies of energy gaps demarcating emerging sixfold braid bands.

The behavior of each arm of the armchair hexagonal ring as a domain similar to the dimerized domains of the trans-polyacetylene has a deeper physical reason, which can be revealed if one considers each arm of the hexagon as a perturbed armchair graphene nanoribbon. Indeed analytic expressions for the energy dispersion of the aGNRs have been recently derived [see Refs. 32(b) and 35]; they have the form

$$
E(k)= \pm\left|t_{1} e^{i k a}+t_{2} e^{-i k b}\right|,
$$

with $k$ being the wave vector along the direction of the edge. $a=a_{0} /(2 \sqrt{3})$ and $b=a_{0} / \sqrt{3}$, with $a_{0}=$ $0.246 \mathrm{~nm}$ being the lattice constant of graphene. $t_{1}=$ $-2 t \cos \left[p \pi /\left(\mathcal{N}_{W}+1\right)\right]+\delta t_{1}, p=1,2, \ldots, \mathcal{N}_{W}$ and $t_{2}=$ $-t+\delta t_{2}$, with $\delta t_{1}, \delta t_{2}$ denoting the perturbation away from a perfect aGNR. The dispersion equation (16) is similar to the tight-binding one describing a onedimensional chain of carbon atoms with bonds (hopping matrix elements) of alternating strength $t_{1}$ and $t_{2}$ (Kekulé structure). The spectrum $E(k)$ exhibits a mass gap $\Delta_{0}=\left|t_{1}-t_{2}\right|$ at $k=\pi /(a+b)$. This behavior is analogous to that of the linear-chain lattice TB model for trans-polyacetylene; see Eq. (2.1) in Ref. 21, In particular, when $t_{1} \neq t_{2}$, Eq. (16) describes a single dimerized domain breaking the $1 \mathrm{D}$ reflectional symme- 
try; when $t_{1}=t_{2}$ (metallic aGNR), it describes a symmetric chain of atoms, which preserves the reflectional symmetry. We note that the factor underlying the formation of dimerized domains in trans-polyacetylene is the Peierls instability incorporated in the Su-SchriefferHeeger model.20|21] The corresponding factor in armchair graphene nanoribbons and rings is topological in nature, i.e., it is a reflection of the lattice distortions of graphene due to the edge termination and the shape.

Further insight can be gained through the observation that the armchair edge by itself reflects the carbon dimerization. Indeed it exhibits shorter dimers (denoted by 1) alternating with longer ones (denoted by 0$)$; see Fig. 1(a). Thus each arm of the graphene ring corresponds to one of two equivalent domains $\ldots, 1,0,1,0, \ldots$ or $\ldots, 0,1,0,1, \ldots$ Following this notation and going around a given corner, one gets symbolically $\ldots, 0,1,0,1,1,0,1,0, \ldots$, i.e., each corner (denoted by an underline) acts as a domain wall separating two alternative domains.

In an infinite polyacetylene chain and at the position of the domain wall, a strongly localized fermionic soliton develops having a fractional charge $\pm 1 / 2$ [19|20 However, the graphene nanoring is a finite system and overall it can carry only integer charges. $\frac{[59] 60]}{}$ Furthermore, the TB results, and their DKP analog in Fig. 7(b), indicate a moderate extent of localization at the corners, resulting from a non-negligible tunneling between the corners. Strong solitonlike localization (exhibiting a $1 / 6$ fractional charge at each corner) can be achieved for larger values of $m_{0}$. Indeed a large $m_{0}$ can localize a massless fermion, as is known from the (theoretical) trapping methodology in $2 \mathrm{D}$ graphene referred to as the infinite-mass boundary condition (introduced in Ref. 61 in the context of trapping neutrinos), as well as from the localization of massless quarks discussed in Ref. 2, The DKP spectrum for a large value $m_{0}=0.30 t / v_{F}^{2}$ is displayed in Fig. 7(c). It is seen that now the single-particle energies falling within the gap $-0.3 t<E<0.3 t$ form horizontal straight lines because the corresponding $\mathrm{AB}$ oscillations have been suppressed due to vanishing of the tunneling between the fermionic solitons at the corners; because of the localization no magnetic flux is trapped by the wave function on the hexagonal ring. It is of interest to note that such a train configuration of fermionic solitons in an hexagonal ring may be referred to as a fractional Wigner crystallite. We note that besides the zero-energy fermionic soliton discussed in the context of the states of polyacetylene, 1920 Fig. 7(c) indicates the emergence of two polaroniclike states 2 with energies $\approx \pm 0.16 t$ falling within the gap.

\section{B. Armchair trigonal ring and constant-mass relativistic regime}

The results of our DKP calculations [associated with Eq. (5)] for the armchair trigonal ring are shown in Fig.
8.

Fig. 8(a) displays the DKP spectra of massless excitations (i.e., $m_{i}^{(n)}=0$ for all $i$ and $n=1,2,3$ ). As was the case with the hexagonal ring [Fig. 7(a)], the massless DKP spectrum in Fig. 8(a) does not exhibit any energy gaps and it is strictly linear and periodic (with period $\Phi_{0}$ ) as a function of $\Phi$ (or equivalently the magnetic field $B)$. We again note that this gapless spectrum remains unchanged even when we consider in addition electrostatic potential steps, $V_{i}^{(n)}>0$, a fact that is a reflection of Klein tunneling. 53154

However, the TB spectrum in Fig. 3(c) exhibits energy gaps (denoted as $\Delta_{0}, \delta_{1}$ and $\delta_{2}$, and highlighted by arrows), which require inclusion of potential barriers in the DKP modeling. Following the analogy with the trans-polyacetylene, it is apparent that the opening of the $\Delta_{0}$ gap indicates the absence of domain alternation. Namely, the armchair trigonal ring represents a realization of a single domain extending along the full length of the triangle. Thus the corners of the triangle act a scatterers instead of domain walls as in the case of the hexagonal ring (see Sec. IV A). Indeed, following the notation of 1 and 0 introduced above for the short and long dimers [Fig. 1 (a)], and going around the corner of the trigonal ring in Fig. 3(a) [or Fig. 3(b)], one gets the sequence $\ldots, 0,0,0,1,1,1,0,0,0, \ldots$, which is in agreement with the presence of the same domain on both sides of the $\pi / 3$ corner. Consequently, in the DKP modeling we keep the same parametrization as in Eqs. (13) and (14), but we replace Eq. 15 by

$$
m_{1}^{(n)}=m_{3}^{(n)}=0, \quad m_{2}^{(n)}=m_{0}=\mathcal{M}, \quad n=1,2,3,
$$

that is, the mass parameters are the same (not alternating) on the three arms of the trigonal ring [see schematic representation in Fig. 6(b)]; recall that $\mathcal{M}$ denotes the fermion rest mass [see discussion below Eq. (5)].

Fig. 8(b) [see also a magnification in Fig. 8(c)] displays the DKP spectrum calculated from the dispersion equation (12) using the above parametrization [see Eqs. [13, (14), and [17)] with $\mathcal{M}=0.02 t / v_{F}^{2}$, and $a=19 a_{0}$, $b=10 a_{0} \cdot 58$ One sees that the DKP spectrum reproduces the two central features of the TB spectrum in Fig. 3(c): (i) the gap $\Delta_{0}$ around $\varepsilon=0$ and (ii) the threefold braid bands which are separated by the gaps $\delta_{1}$ and $\delta_{2}$ at higher energies.

\section{Zigzag hexagonal ring and constant-mass nonrelativistic limit}

As aforementioned, the TB spectrum of the hexagonal graphene ring with zigzag edges [Fig. 4(b)] shows trends associated with a large-mass fermion in the nonrelativistic regime: (i) almost-perfect parabolic $B$-dependence and (ii) energy gaps $\delta_{0}, \delta_{1}$ and $\delta_{2}$ that are as large as the width of the braid bands. We remind that, for a massless excitation (ultrarelativistic limit), the spectra 
(a)

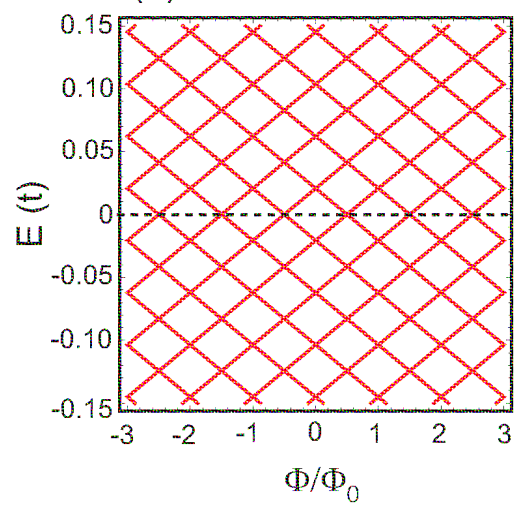

(b)

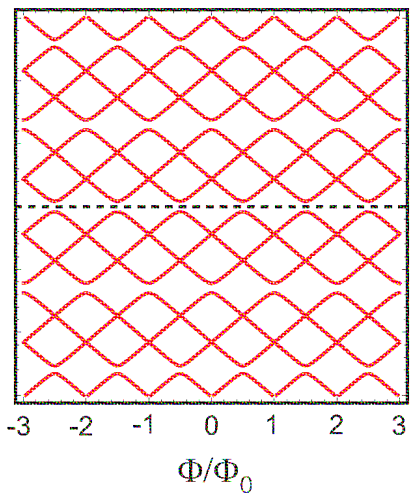

(c)

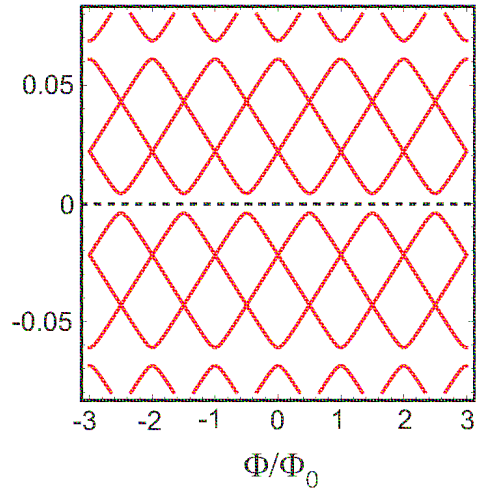

FIG. 8. (Color online) Spectra from the DKP model (relativistic regime) corresponding to the schematic case (triangle) in Fig. 6(b); see text and Eq. 17) for the full set of parameters employed. Panels (a) and (b,c) correspond to different choices of $m_{0}$ [denoted also as $\mathcal{M}$ here; see Eq. [17] ]. (a) $m_{0}=0$ and any $V_{i}$. Note the absence of band gaps due to the Klein paradox. (b,c) $m_{0}=0.02 t / v_{F}^{2}$, and in (c) we display a magnification of the region around $E=0$. Note the similarity of the spectrum with that of the armchair graphene ring in Fig. 3(c). The dashed line indicates the energy zero.

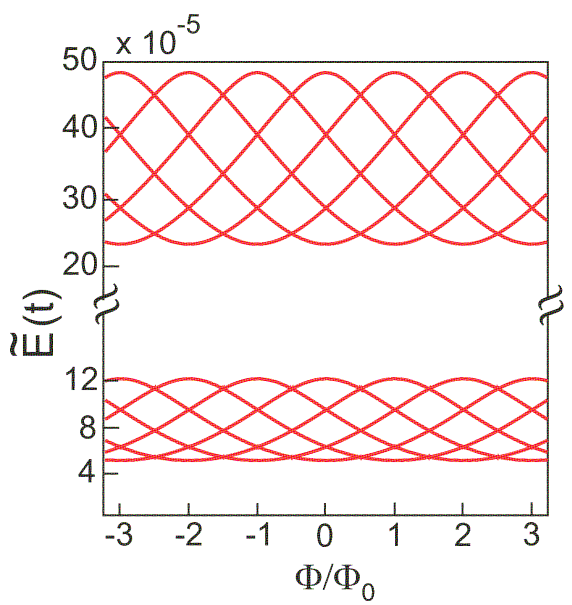

FIG. 9. (Color online) Spectra from the DKP model (nonrelativistic regime) corresponding to the schematic case (hexagon) in Fig. 6(c); see text and Eqs. (18,20) for the full set of parameters employed. The energies do not contain the restmass contribution, $\frac{63}{6}$ that is, $\widetilde{E}(t)=E-\mathcal{M} v_{F}^{2}$, where $E$ is the value obtained from solution of the DKP model and $\mathcal{M}$ is determined by fitting $\widetilde{E}$ to the TB results [Fig. 4(b)]. The rest mass determined to yield the best fit shown here, is given by $\mathcal{M}=42.06 t / v_{F}^{2}$. This mass is heavier than that of the electron $\left(2.10 t / v_{F}^{2}\right)$, suggesting an analogy with leptons.

are strictly linear and exhibit no gaps [see Figs. 7(a) and 8(a)]; consequently small or moderate mass terms (relativistic regime) will result in smaller energy gaps compared to the width of the braid bands [see Fig. 7(b) and Figs. 8(b) and 8(c)].

The sharply different physics (nonrelativistic versus relativistic behavior) underlying the $\mathrm{TB}$ spectra of the zigzag and armchair rings originates from the different edge topology. Following the notation of 1 and 0 introduced above for the short and long dimers [Fig. 1(a)], and going around the $2 \pi / 3$ corner of the zigzag ring in Fig. $4(\mathrm{a})$, one further gets the sequence $\ldots, 0,0,0, \underline{1}, 0,0,0, \ldots$ This, in addition to the anticipation of a nonrelativistic regime, suggests that the entire hexagonal zigzag ring constitutes a single domain with embedded (corner) impurities (electrostatic potential scatterers).

In light of the above, using a large rest mass $\mathcal{M}$ in Eq. (9) [nonrelativistic limit, see Eqs. (4.27) and (4.28) in Ref. 2], we were able to reproduce in the DKP model [see Fig. 9 the overall trends of the TB spectrum [Fig. 4(b)] for the zigzag ring. The DKP parameters used 58 were as follows, Lengths:

$$
L_{1}^{(n)}=L_{3}^{(n)}=a=1.5 a_{0}, \quad L_{2}^{(n)}=b=28 a_{0} .
$$

Rest mass:

$$
\mathcal{M}=m_{1}^{(n)}=m_{2}^{(n)}=m_{3}^{(n)}=42.06 t / v_{F}^{2} .
$$

Potential barrier step at each corner:

$$
V=V_{1}^{(n)}=V_{3}^{(n)}=80 \times 10^{-5} t, \text { with } V_{2}^{(n)}=0 .
$$

A schematic representation of these parameters is given in Fig. 6(c).

In the evaluation of the nonrelativistic limit of the DKP model, one often sets $E=\mathcal{M} v_{F}^{2}+\widetilde{E}$ for the positive energies [see Eq. (4.27) in Ref. 2]. The quantity calculated from the DKP model in this limit is $E$, while in comparing with the TB results [Fig. 4 (b)] we plot in Fig. $9 \widetilde{E}$ vs. $\Phi$. The value of $\mathcal{M}$ is determined by finding the best fit to the TB results. ${ }^{63}$ It is expected that further improvement in the agreement between the TB and DKP approaches can be achieved by employing smooth (rather than square-shaped) profiles for the potential barriers. The constant mass, $\mathcal{M}$, found by us here is twenty times larger than the rest mass of the electron, indicating an analogy with electronlike leptons, ${ }^{23}$ rather than the electron itself. 
We note that the large rest mass $\mathcal{M}$ found in this section is unrelated to the energy gap $\delta_{0}$ [see Fig. 4(b)]; $\left(\mathcal{M} v_{F}^{2} / \delta_{0} \sim 10^{5}\right)$. Indeed in the nonrelativistic regime, the effective mass is related to the second derivative of the band spectra.$\frac{48}{}$ Our findings concerning the zigzag graphene ring are in agreement with the analysis of Ref. 48 regarding the TB spectra of (infinite) symmetric polyacene, which is a single chain of fused benzene rings and can be considered as the thinnest possible zigzag graphene nanoribbon.

\section{THE UNDERLYING RELATIVISTIC QUANTUM FIELD LAGRANGIAN}

In the above (see Sec. II), we identified characteristic patterns of tight-binding spectra in planar graphene nanorings and we described their dependence on the edge termination (armchair versus zigzag) and the ring shape (hexagonal versus trigonal). Subsequently, we presented a unified interpretation of the TB results using a DiracKronig-Penney model (Sec. III), built upon the generalized 1D Dirac equation [Eq. (5)]. A central finding of our relativistic DKP analysis were the close analogies (found in Secs. IV A and IV B between the behavior of armchair hexagonal and trigonal graphene nanorings and the physics of trans-polyacetylene 192

A natural next step towards a deeper understanding of the connection of our findings to relativistic quantum field theory is the elucidation of the underlying Lagrangian formalism. Motivated by the theory of transpolyacetylene, ${ }^{20}$ we write a total Lagrangian density

$$
\mathcal{L}=\mathcal{L}_{f}+\mathcal{L}_{\phi},
$$

which is the sum of (partial) Lagrangian densities for the fermion field $\Psi$ [Eq. (6)] and the bosonic scalar field $\phi(x)$. We note that the scalar field $\phi(x)$ was denoted earlier also as $m(x)$ and was referred to as a position-dependent mass; see Sec. III and Sec. IV A.

The stationary generalized Dirac equation [Eq. (5)] can be derived from the fermionic Lagrangian density

$$
\mathcal{L}_{f}=-i \hbar \Psi^{\dagger} \frac{\partial}{\partial t} \Psi-i \hbar v_{F} \Psi^{\dagger} \alpha \frac{\partial}{\partial x} \Psi-\phi \Psi^{\dagger} \beta \Psi,
$$

where we have neglected contributions from the electrostatic potentials (see discussion in Sec. IV A).

In Eq. (22), the last term

$$
\mathcal{L}_{Y}=-\phi \Psi^{\dagger} \beta \Psi,
$$

which depends on both the fermion $\Psi$ and scalar $\phi$ fields, has the form of a Yukawa coupling. $\mathcal{L}_{Y}$ is the potential agent for rest-mass acquisition by the originally massless fermion [described by the first two terms in the right hand side of Eq. [22] ].

The Yukawa interaction is also used in the Standard Mode $\sqrt{64} \sqrt[66]{6}$ to describe the coupling between the Higgs field and the massless quark and lepton fields (i.e., the fundamental fermion particles). Through spontaneous symmetry breaking $\frac{\sqrt{67} 70}{70}$ of the Higgs field [which is a complex $S U(2)$ doublet of four real scalar fields $\phi]$, these fermions acquire a mass proportional to the vacuum expectation value of the Higgs field ${ }^{71}$.73

The essential observation that we make here is that, although, due to the $1 \mathrm{D}$ character of the graphene rings, the 1D Lagrangian in Eq. 21) does not possess the full richness of the Lagrangian of the Higgs sector in the Standard Model, both share the central aspect of symmetry breaking and mass acquisition by a fermion via a Yukawatype interaction.

We turn next to the task of constructing the Lagrangian part $\mathcal{L}_{\phi}$ for the scalar field $\phi$, which is of the general form ${ }^{74 \mid 75}$ (in $1+1$ dimensions, i.e., time plus one space dimension)

$$
\mathcal{L}_{\phi}=-\frac{1}{2}\left(\frac{\partial \phi}{\partial x}\right)^{2}-V(\phi),
$$

and which preserves the reflectional $Z_{2}$ symmetry, i.e., it is invariant under $\phi \rightarrow-\phi$. Note that we are interested in the adiabatic approximation, and thus we omit the time dependent terms in $\mathcal{L}_{\phi}$.

The emergence of a constant mass for the armchair trigonal ring (Sec. IVB) could simply be accounted for by considering a constant value of $\phi=\phi_{0}=\mathcal{M}$ in the fermion Lagrangian $\mathcal{L}_{f}$; then one needs to pay no further consideration to the bosonic $\mathcal{L}_{\phi}$. However, a more general position-dependent field, $\phi(x)=m(x)$, was found to be essential in our DKP-model analysis of the armchair hexagonal ring (Sec. IV A). In this case, $\phi(x)$ alternates between two unequal values $\pm \phi_{0}$, with $\phi_{0}=m_{0}$ [see Fig. 6(a)]. This indicates breaking of the $Z_{2}$ symmetry of the solutions to the equation of motion derived from the Lagrangian in Eq. (24). An expression for $V(\phi)$ which reproduces qualitatively the above behavior (including the trigonal ring case) is the socalled $\phi^{4}$, which corresponds to a quartic double well potential in $\phi$, i.e.,

$$
V(\phi)=\frac{\xi}{4}\left(\phi^{2}-\zeta^{2}\right)^{2},
$$

where $\xi$ and $\zeta$ are parameters.

With the potential in Eq. 25), the bosonic sector has the field equation (see Ch. 2.3 in Ref. 74 and Ch. 1.1 in Ref. 75)

$$
-\frac{\partial^{2} \phi}{\partial x^{2}}+\xi\left(\phi^{2}-\zeta^{2}\right) \phi=0 .
$$

Two solutions of Eq. (26) are $\phi(x)= \pm \phi_{0}= \pm \zeta$; these solutions break the symmetry since $\phi_{0} \neq 0$. Using these solutions in the Dirac Eq. (5), one obtains the standard constant-mass Dirac equation 1 for the fermionic field. This case corresponds to the behavior of the armchair trigonal ring (Sec. IV B), as well as to that of the zigzag hexagonal ring (Sec. IV C).

In addition, however, Eq. (26) has nonlinear solutions that interpolate between the locations $\phi_{0}$ and $-\phi_{0}$ of the 


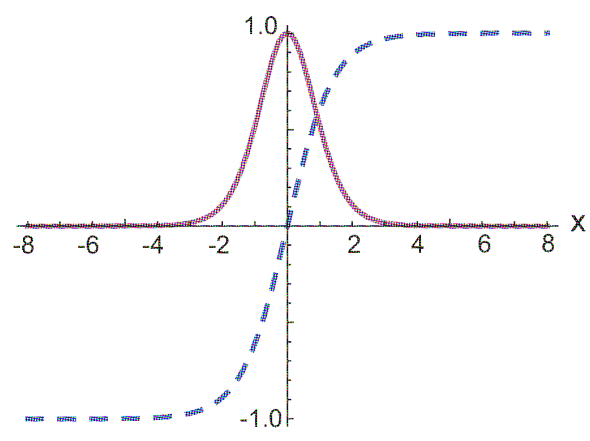

FIG. 10. (Color online) Dashed line: The scalar $Z_{2}$ kink soliton [Eq. (27)]. Solid line: The particle density (unnormalized) of the corresponding enslaved fermionic soliton [Eq. [29]]. The values $\xi=1$ and $\zeta=1$ were used. The domain wall is located at $x=0$.

two minima of the $V(\phi)$ potential. One of these nonlinear solutions is called the $Z_{2}$ kink soliton and the other the $Z_{2}$ antikink soliton. The kink soliton is given by

$$
\phi_{k}(x)=\zeta \tanh \left(\sqrt{\frac{\xi}{2}} \zeta x\right),
$$

and the antikink soliton has the form

$$
\bar{\phi}_{k}(x)=-\phi_{k}(x) .
$$

We note that $\phi_{k}( \pm \infty)= \pm \zeta$ and $\bar{\phi}_{k}( \pm \infty)=\mp \zeta$, while $\phi_{k}(0)=\bar{\phi}_{k}(0)=0$.

Using these kink or antikink scalar fields, the corresponding generalized Dirac equation [Eq. (50)] possesses fermionic solitor ${ }^{20}$ solutions of the form (here $\alpha=\sigma_{2}$, $\left.\beta=\sigma_{1}\right)$

$$
\Psi_{S}(x) \propto\left(\begin{array}{c}
\exp \left(-\int_{0}^{x} \phi_{k}\left(x^{\prime}\right) d x^{\prime}\right) \\
0
\end{array}\right) .
$$

The importance of these fermionic solitons lies in the fact that they have strictly zero energies $\stackrel{20}{20}$ thus they fall into the particle/antiparticle (valence/conductance band) energy gap. Furthermore, they are localized at $x=0$, which is the domain wall between $x>0\left(\phi_{0}\right)$ and $x<0\left(-\phi_{0}\right)$. The enslavement of the fermionic soliton $\Psi_{S}(x)$ by the scalar potential of the kink soliton $\phi_{k}(x)$ is evident from Eq. (29). This is also reflected by the localization of $\Psi_{S}(x)$ on the domain wall $x=0$ (see Fig. 10 .

It is apparent that such zero-mode fermionic solitons $\Psi_{S}(x)$ underlie qualitatively the behavior of the fermion excitations in the armchair hexagonal graphene nanoring (Sec. IV A), with the corners of the ring behaving as domain walls and the $m(x)$ stepwise function in the DKP model $\left[\phi(x)\right.$ in Eq. (5)] mimicking an alternation of $Z_{2}$ kink and antikink scalar solitons [Eqs. (27) and (28)]; for a quantum-field-theory description of a train of alternating kinks and antikinks, see Ch. 1.7 in Ref. 75.

\section{CONCLUSIONS}

The paper investigated the different behavior of the Aharonov-Bohm spectra and magnetic-field induced oscillations for three characteristic cases of planar graphene nanorings, i.e., an hexagonal ring with armchair edge terminations, a trigonal ring with armchair edge terminations, and an hexagonal ring with zigzag edge terminations. The tight-binding results (Sec. III) were analyzed with the help of a $1 \mathrm{D}$ relativistic Dirac-KronigPenney model ${ }^{2}$ (Sec. III), which accounts for the virtual superlattice associated $\frac{18}{18}$ with the applied magnetic field. This analysis revealed unexpected topological effects and condensed-matter analogies with elementary particle physics.

In particular, the behavior found by us for the armchair hexagonal ring (Sec. IV A ) is reminiscent of the extreme relativistic regime describing zero-energy fermionic solitons with fractional charge in quantum field theory $\sqrt{37}$ and in the theory of trans-polyacetylene $\frac{19}{22}$ This regime results from a consideration of a modified (generalized) Dirac equation with a position-dependent mass term (or equivalently a position-dependent scalar bosonic field). In contrast, the quasiparticle excitations in the armchair trigonal ring (Sec. IV B) behave as relativistic Dirac fermions having a constant mass. A unification of these two dissimilar behaviors was presented in Sec. $\mathrm{V}$ by introducing the underlying relativistic Lagrangian formalism for a fermionic and a scalar bosonic fields coupled via a Yukawa interaction. The Yukawa term in conjunction with the breaking of the $Z_{2}$ reflectional symmetry of the scalar field may result in two outcomes, i.e., formation of a fermionic soliton (armchair hexagonal ring) or mass generation (armchair trigonal ring). The profoundly differing behaviors found by us for the armchair hexagonal and trigonal rings (with both sharing similar spatial dimensions), are manifestations of the quantum topological nature of this behavior, as distinguished from "quantum size effects" which are length-scale dependent phenomena, originating from spatial confinement of the electrons (quasiparticles, in general) $\frac{76+79}{7}$

The behavior of the zigzag hexagonal ring resembles the low-kinetic-energy nonrelativistic regime of a leptonlike fermion having a rest mass larger than that of the electron (Sec. IV C). This behavior contrasts with the relativistic ones found for the aforementioned armchair rings, thus highlighting the compounded topological and edge -termination effects.

These findings ${ }^{80181}$ highlight the potential of graphene nanosystems for providing a bridge between condensedmatter and particle physics, well beyond the paradigm of the massless neutrinolike fermion familiar from the 2D graphene sheet. Furthermore beyond the realm of graphene proper, where atomically precise narrow nanoribbons have already been synthesized ${ }^{36}$ we anticipate that our theoretical predictions could be tested using an ever expanding class of designer-Dirac-fermion manmade systems, such as optical lattices comprising ul- 
tracold atoms, $10[13$ or "molecular" 12 and nanopatterned artificial graphene.

\section{ACKNOWLEDGMENTS}

This work was supported by the Office of Basic Energy Sciences of the US D.O.E. under contract FG05-
86ER45234.
Igor.Romanovsky@physics.gatech.edu

† Constantine.Yannouleas@physics.gatech.edu

‡ Uzi.Landman@physics.gatech.edu

1 P. A. M. Dirac, Proc. Roy. Soc. London, Ser. A 117, 610 (1928).

2 B. H. J. McKellar and G. J. Stephenson, Jr., Phys. Rev. C 35, 2262 (1987).

3 J. D. Björken and S. D. Drell, Relativistic quantum mechanics (New York, McGraw-Hill, 1964).

4 D. Griffiths, Introduction to Elementary Particles (WileyVCH, Weinheim, 2008).

${ }^{5}$ K. S. Novoselov, A. K. Geim, S. V. Morozov, D. Jiang, Y. Zhang, S. V. Dubonos, I. V. Grigorieva, and A. A. Firsov, Science 306, 666 (2004).

${ }^{6}$ P. R. Wallace, Phys. Rev. 71, 622 (1947).

7 A. H. Castro Neto, F. Guinea, N. M. R. Peres, K. S. Novoselov, and A. K. Geim, Rev. Mod. Phys. 81, 109 (2009).

8 H. Weyl, Z. Phys. 56, 330 (1929) [English translation: Surveys High Energ. Phys. 5, 261 (1986)].

9 S.-L. Zhu, B. Wang, and L.-M. Duan, Phys. Rev. Lett. 98, 260402 (2007).

10 J. Ruostekoski, J. Javanainen, and G. V. Dunne, Phys. Rev. A 77, 013603 (2008).

11 Y.L. Chen et al., Science 329, 659 (2010).

12 K. K. Gomes, W. Mar, W. Ko, F. Guinea, H. C. Manoharan, Nature 483, 306 (2012).

13 D.-W. Zhang, L.-B. Shao, Z.-Y. Xue, H. Yan, Z. D. Wang, S.-L. Zhu, arXiv:1206.5614.

14 I. Romanovsky, C. Yannouleas, and U. Landman, Phys. Rev. B 86, 165440 (2012).

15 I. Romanovsky, C. Yannouleas, and U. Landman, Phys. Rev. B 85, 165434 (2012).

16 Y. Aharonov and D. Bohm, Phys. Rev. 115, 485 (1959).

17 For the original nonrelativistic model, see R. de L. Kronig and W. G. Penney, Proc. R. Soc. London, Ser. A 130, 499 (1931).

18 M. Büttiker, Y. Imry, and R. Landauer, Phys. Lett. A 96, 365 (1983).

19 R. Jackiw, Phys. Scr. T146, 014005 (2012).

20 R. Jackiw and J. R. Schrieffer, Nucl. Phys. B 190, 253 (1981).

21 A. J. Heeger, S. Kivelson, J. R. Schrieffer, and W.-P. Su, Rev. Mod. Phys. 60, 781 (1988).

22 D. K. Campbell, Synth. Met. 125, 117 (2001).

${ }^{23}$ F. Halzen and A. D. Martin, Quarks and leptons: an introductory course in modern particle physics (New York, Wiley, 1984).

${ }^{24}$ P. Recher, B. Trauzettel, A. Rycerz, Y. M. Blanter, C. W. J. Beenakker, and A. F. Morpurgo, Phys. Rev. B 76,
235404 (2007).

25 D. A. Bahamon, A. L. C. Pereira, and P. A. Schulz, Phys. Rev. B 79, 125414 (2009).

26 T. Luo, A. Iyengar, A. H. Fertig, and L. Brey, Phys. Rev. B 80, 165310 (2009).

27 H. A. Fertig and L. Brey, Phil. Trans. R. Soc. A 368, 5483 (2010).

28 M. M. Ma and J. W. Ding, Solid State Commun. 150, 1196 (2010)

29 D. S. L. Abergel, V. M. Apalkov, and T. Chakraborty, Phys. Rev. B 78, 193405 (2008).

30 M. Zarenia, J. M. Pereira, A. Chaves, F. M. Peeters, and G. A. Farias, Phys. Rev. B 81, 045431 (2010).

31 J. Schelter, P. Recher, and B. Trauzettel, Sol. State Commun. 152, 1411 (2012).

32 For TB and related studies, see for example (a) K. Nakada, M. Fujita, G. Dresselhaus, and M. S. Dresselhaus, Phys. Rev. B 54, 17954 (1996); (b) H. Zheng, Z. F. Wang, T. Luo,Q. W. Shi, and J. Chen, Phys. Rev. B 75, 165414 (2007). (c) Alexander Onipko, Phys. Rev. B 78, 245412 (2008).

33 For density-functional-theory studies, see for example Y.W. Son, M. L. Cohen, and S. G. Louie, Phys. Rev. Lett. 97, 216803 (2006).

34 For continuum DW studies, see for example (a) L. Brey and H. A. Fertig, Phys. Rev. B 73, 235411 (2006); (b) L. Brey and H. A. Fertig, Phys. Rev. B 73, 195408 (2006).

35 For a recent theoretical review, see K. Wakabayashi, K.I. Sasaki, T. Nakanishi, and T. Enoki, Sci. Technol. Adv. Mater. 11, 054504 (2010).

36 See for example J.-M. Cai, P. Ruffieux, R. Jaafar et al., Nature 466, 470 (2010)

37 R. Jackiw and C. Rebbi, Phys. Rev. D 13, 3398 (1976).

38 Carbon nanotubes (CNTs) are yet another extensively studied $1 \mathrm{D}$ carbon allotrope, which also exhibit a $\Delta_{0}$ gap at the Fermi level [see reviews: T. Ando, J. Phys. Soc. Jpn. 74, 777 (2005); J.-Ch. Charlier, X. Blase, and S. Roche, Rev. Mod. Phys. 79, 677 (2007)]. The $\Delta_{0}$ gap in CNTs, however, is due to a finite quantum-size effect [see Sec. 3.2 in the T. Ando review], namely to a quantization of the free-electron motion along the circumference of the nanotube. CNTs do not seem to be susceptible to a spontaneous Peierls transition either [R. Saito, M. Fujita, G. Dresselhaus, and M. S. Dresselhaus, Appl. Phys. Lett. 60, 2204 (1992)]. However, this subject remains still open to date. A most recent study suggests that a Peierls transition may be possible in narrow CNTs; see G. Dumont, P. Boulanger, M. Côté, and M. Ernzerhof, Phys. Rev. B 82, 035419 (2010).

39 F. Englert and R. Brout, Phys. Rev. Lett. 13, 321 (1964). 
40 P. W. Higgs, Phys. Rev. Lett. 13, 508 (1964).

41 G. S. Guralnik, C. R. Hagen, and T. W. B. Kibble, Phys. Rev. Lett. 13, 585 (1964).

42 P. W. Anderson, Phys. Rev. 130, 439 (1962).

43 T. Sato, K. Segawa, K. Kosaka, S. Souma, K. Nakayama, K. Eto, T. Minami, Y. Ando, and T. Takahashi, Nature Phys. 7, 840 (2011).

44 M. Gibertini, A. Singha, V. Pellegrini et al., Phys. Rev. B 79, 241406 (2009).

45 Study cases of armchair graphene rings with arms corresponding to the other two classes of ideal aGNRs (namely with $\mathcal{N}_{W}=3 l-2$ and $\mathcal{N}_{W}=3 l, l=1,2,3, \ldots$ ) will be presented in a future publication [I. Romanovsky, C. Yannouleas, and U. Landman, in preparation].

${ }^{46}$ R. B. Lehoucq, D. C. Sorensen, and C. Yang, ARPACK Users' Guide: Solution of Large-Scale Eigenvalue Problems with Implicitly Restarted Arnoldi Methods (SIAM, Philadelphia, 1998).

47 H.-F. Cheung, Y. Gefen, E. K. Riedel, and W.-H. Shih, Phys. Rev. B 37, 6050 (1988).

48 S. Kivelson and O. L. Chapman, Phys. Rev. B 28, 7236 (1983).

49 See, e.g., Appendix A in H. Nitta, T. Kudo, and H. Minowa, Am. J. Phys. 67, 966 (1999).

50 See, e.g., Sec. II in Ref. 2 and A. Sánchez, E. Maciá, and F. Domínguez-Adame, Phys. Rev. B 49, 147 (1994).

${ }^{51}$ See also R. Gilmore, Elementary Quantum Mechanics in One Dimension (The Johns Hopkins University Press, Baltimore, 2004), Chaps. 37 and 38.

52 I.I. Cotaescu and E. Papp, J. Phys.: Condens. Matter 19, 242206 (2007)

53 O. Klein, Z. Phys. 53, 157 (1929).

54 M. I. Katsnelson, K. S. Novoselov, and A. K. Geim, Nat. Phys. 2, 620 (2006).

55 C.-H. Park, L. Yang, Y.-W. Son, M. L. Cohen, and S. G. Louie, Phys. Rev. Lett. 101, 126804 (2008).

56 M. Barbier, F. M. Peeters, P. Vasilopoulos, and J. M. Pereira, Jr., Phys. Rev. B 77, 115446 (2008).

57 D. P. Arovas, L. Brey, H. A. Fertig, E.-A. Kim, and K. Ziegler, New J. Phys. 12, 123020 (2010).

58 The total length $L=2 a+b$ equals approximately that of the side of the corresponding graphene ring.

59 R. Rajaraman and J. S. Bell, Phys. Lett. B 116, 151 (1982).

60 S. Kivelson and J. R. Schrieffer, Phys. Rev. B 25, 6447 (1982).

61 M. V. Berry and R. J. Mondragon, Proc. R. Soc. London, Ser. A 412, 53 (1987).

62 D. K. Campbell, A. R. Bishop, and K. Fesser, Phys. Rev. B 26, 6862 (1982).

${ }^{63}$ In the nonrelativistic limit, for negative energies (representing holes with $-e$ charge), one sets $E=-\mathcal{M} v_{F}^{2}+\widetilde{E}$ and uses a negative potential step $-V$. The resulting spectrum is mirror-symmetric about the horizontal axis $\widetilde{E}=0$; note the we mentioned here holes rather than antiparticles.

64 S. L. Glashow, Nucl. Phys. 22, 579 (1961).

65 S. Weinberg, Phys. Rev. Lett. 19, 1264 (1967).

66 A. Salam, Elementary Particle Physics: Relativistic Groups and Analyticity, Eighth Nobel Symposium Stockholm, edited by N. Svartholm (Almquvist and Wiksell, Stockholm, 1968) p 367.

67 Symmetry breaking in elementary particle physics was introduced in Ref. 68. For the importance of symmetry breaking in bulk condensed-matter systems, see Ref. 69 For symmetry breaking and the subsequent step of symmetry restoration in finite systems, see Ref. 70 .

68 Y. Nambu, Phys. Rev. Lett. 4, 380 (1960); Y. Nambu and G. Jona-Lasinio, Phys. Rev. 124, 246 (1961); Phys. Rev. 122, 345 (1961).

69 P. W. Anderson, Science 177393 (1972); Basic Notions of Condensed Matter Physics (Addison-Wesley, Reading, 1984).

70 C. Yannouleas and U. Landman, Rep. Prog. Phys. 70, 2067 (2007)

71 For a pedagogical introduction, proceeding step-by-step from the simplest example to the full Higgs mechanism of mass generation in the Standard Model, see Refs. 72 and 73

72 V. A. Bednyakov, N. D. Giokaris, and A. V. Bednyakov, Phys. Part. Nucl. 39, 13 (2008); arXiv:hep-ph/0703280.

73 D. McMahon, Quantum Field Theory DeMystified (McGraw-Hill, New York, 2008).

74 R. Rajaraman, Solitons and Instantons: An Introduction to Solitons and Instantons in Quantum Field Theory (North-Holland, Amsterdam, 1982).

75 T. Vachaspati, Kinks and Domain Walls: An Introduction to Classical and Quantum Solitons (Cambridge University Press, Cambridge, 2006).

76 For a review of quantum size effects of electrons confined in ultrathin metallic films see: M. C. Tringides, M. Jalochowski, and E. Bauer, Phys. Today 60, 50 (2007).

77 For a review of quantum size effects before the discovery [Ref. [8(a)] of electronic shell effects in clusters, see: W. P. Halperin, Rev. Mod. Phys. 58, 533 (1986).

78 For electronic shell effects in clusters, see (a) W. D. Knight, K. Clemenger, W. A. De Heer, W. A. Saunders, M. Y. Chou, and M. L. Cohen, Phys. Rev. Lett. 52, 2141 (1984); (b) W. A. De Heer, Rev. Mod. Phys. 65, 611 (1993); (c) C. Yannouleas and U. Landman, in Large Clusters of Atoms and Molecules, edited by T. P. Martin (Kluwer, Dordrecht, 1996) p. 131

79 For electronic shell effects in 2D constrictions and in 3D quantum wires, see: (a) B. J. van Wees, H. van Houten, C. W. J. Beenakker, J. G. Williamson, L. P. Kouwenhoven, D. van der Marel, and C. T. Foxton, Phys. Rev. Lett. 60, 646 (1988); (b) J. I. Pascual, J. Mendez, J. Gomez-Herrero, A. M. Baro, N. Garcia, U. Landman, W. D. Luedtke, E. N. Bogachek, and H.-P. Cheng, Science 267, 1783 (1995); (c) C. Yannouleas, E. N. Bogachek, and U. Landman, Phys. Rev. B 57, 4872 (1998); (d) N. Agrait, A. L. Yeyati, and J. M. van Ruitenbeek, Phys. Reps. 377, 81 (2003).

${ }^{80}$ For simplicity, in describing the quantum-field-theory analogies in Section $V$, the scalar field $\phi(x)=m(x)$ associated with the armchair trigonal case [Fig. 6(b)] was treated as a constant mass along the full perimeter of the triangle. Although this is a reasonable zero-order approximation, as it is evident from Fig. 6(b), the $\phi(x)$ field exhibits depressions (it attains zero values) at the corners of the triangle. Properly speaking, such a scalar-field dependence leads to states that are not identical with free Dirac particles. In the case of conducting polymers, the corresponding states are known as polarons [see Refs. 21, 22, and 62, and S. Brazovskii and N. N. Kirova, Zh. Eksp. Teor. Fiz. Pis'ma 33, 6 (1981) [JETP Lett. 33, 4 (1981)]. In particle physics, the similar states are referred to as fermion bags [see, e.g., D. K. Campbell and Y.-T. Liao, Phys. Rev. D 14, 2093 (1976); R. F. Dashen, B. Hasslacher, and A. Neveu, Phys. 
Rev. D 12, 2443 (1975)], and they correspond to mass acquisition processes that are in principal more general than the Higgs mechanism [see R. MacKenzie and W. F. Palmer, Phys. Rev. D 42, 701 (1990)]. The polaron states provide also a connection [see Ref. 22 to the self-consistent GrossNeveu model [D. J. Gross and A. Neveu, Phys. Rev. D 10, 3235 (1974)]. Such aspects, as they relate to the planar graphene rings, will be elaborated in a future publication [see Ref. 45].

81 The general particle-physics and quantum-field theory analogies, discussed in this paper regarding the occurrence or absence of an energy gap at the Fermi level of graphene-based nanostructures, may also provide a unifying theoretical background for the gap behavior in the moiré superlattices formed in heterostructures created by placing a graphene monolayer on a hexagonal boron nitride $(\mathrm{hBN})$ substrate. For the latest developments in this area, see, e.g., the following eprints (noticed at the proof-reading stage of this paper): B. Hunt, J. D. Sanchez-Yamagishi, A. F. Young, K. Watanabe, T. Taniguchi, P. Moon, M. Koshino, P. Jarillo-Herrero, and R. C. Ashoori, arXiv:1303.6942 and J. R. Wallbank, A. A. Patel, M. Mucha-Kruczynski, A. K. Geim, and V. I. Fal'ko, arXiv:1211.4711 